\title{
Medullary haemorrhage: a report of two cases
}

\author{
F. L. MASTAGLIA, ${ }^{1}$ B. EDIS, AND B. A. KAKULAS \\ From the Department of Pathology, University of Western Australia, and the Neuropathology \\ Laboratory, Royal Perth Hospital
}

The medulla oblongata is rarely the site of primary parenchymal haemorrhage. Pontine haemorrhages, whether primary or secondary to tentorial herniation, likewise rarely extend into the medulla. In a review of 105 cases of cerebral haemorrhage Mutlu, Berry, and Alpers (1963) commented that medullary haemorrhages were too small and too infrequently encountered to warrant analysis. Pezzani and D'Alessio (1956) found petechial haemorrhages in the medulla of many fatal cases of foetal anoxia, and Gross and Ehrlich (1940) found medullary haemorrhages in a small number of cases of severe head injury. We have found reference to five other cases of medullary haemorrhage in the literature. Two of these were due to rupture of angiomatous malformations (Bergman, 1950; Bosch and Janssen, 1962), while the other three were spontaneous (Barron and Fergusson, 1959; Façon, Schwarz, and Ionescu, 1962).

In view of the rarity of this condition, the following two cases seen at the Royal Perth Hospital in the past six years are reported. In one case the haemorrhage was primarily medullary, while in the other it extended into the medulla from the pons.

\section{CASE 1}

The patient was a 48-year-old woman who was awakened by severe left-sided headache and subsequently vomited. There was a past history of hypertension, which 14 years previously had been treated by lumbar sympathectomy with partial improvement. An arthrodesis of the left knee had been performed eight years previously because of severe osteoarthritis. Over the preceding year she had suffered from angina pectoris which had increased in severity over the previous two months. Oral anticoagulant therapy had been commenced and she was having a maintenance dose of phenindione, $75 \mathrm{mg}$ daily. The prothrombin time nine days before the ictus was 25 seconds (control 13 seconds).

The headache improved considerably after an intramuscular injection of pethidine, but she subsequently complained of numbness over the right eye. The headache had returned by the following morning and was once

'Present address: Regional Neurological Centre, The General Hospital, Newcastle upon Tyne, Great Britain. again associated with vomiting. She was fully conscious and neurological examination revealed inco-ordination in the left upper limb, unawareness of the position of the left arm and leg, absent reflexes in the left upper limb, and an absent left plantar response. On the following day she was fully conscious and oriented and speech was normal. The visual fields were intact. Fundoscopy revealed tortuosity and irregular narrowing of retinal vessels. The pupils were equal and reacted briskly to light and on accommodation. There was no diplopia or nystagmus. Corneal and facial sensation, facial movements, and hearing were normal as were palatal, pharyngeal, and lingual sensation and movements. There was slight reduction in muscle power in the left arm and leg. Inco-ordination in the left upper limb and impaired appreciation of the position of the left arm and leg in space were still present. The reflexes were exaggerated in the left upper limb and at the left ankle and there was a Babinski response on the left side. The superficial abdominal reflexes were absent on both sides. Vibration sense was reduced on the left side of the body. There was normal appreciation of light touch, pain, and temperature, but superficial stimuli elicited unpleasant dysaesthesiae in the left upper limb. Although the patient complained of numbness on the right side of the body, there was no objective evidence of sensory loss on the right side. The patient was afebrile; the pulse rate was 72 per minute; blood pressure $170 / 100$. No abnormalities were detected on general examination. The patient died suddenly on the following day.

NECROPSY REPORT The necropsy (A62.225) was performed by Professor R. E. J. ten Seldam. The heart weighed $290 \mathrm{~g}$. There was left ventricular hypertrophy with patchy interstitial and sub-endocardial fibrosis. Atherosclerosis was particularly severe in the aorta and in the descending branch of the left coronary artery. The kidneys were normal in weight $(128,132 \mathrm{~g})$, but were finely granular and microscopic examination showed the changes of benign hypertensive arteriolosclerosis. Other findings included lymphocytic thyroiditis and cystic mastopathy of the breasts.

NEUROPATHOLOGICAL FINDINGS A recent haemorrhage was present in the medulla oblongata and extended into the upper cervical cord (Fig. 1). Rostrally the haemorrhage extended as far as the inferior olivary nucleus where it measured $4 \times 2 \mathrm{~mm}$ and involved the gracile and cuneate nuclei. Below this level it was larger and 
involved the deeper structures on the left side of the medulla. It reached a maximum size of $6 \mathrm{~mm}$ at the junction of medulla and cervical cord where it was more central in position and occupied almost the whole of the

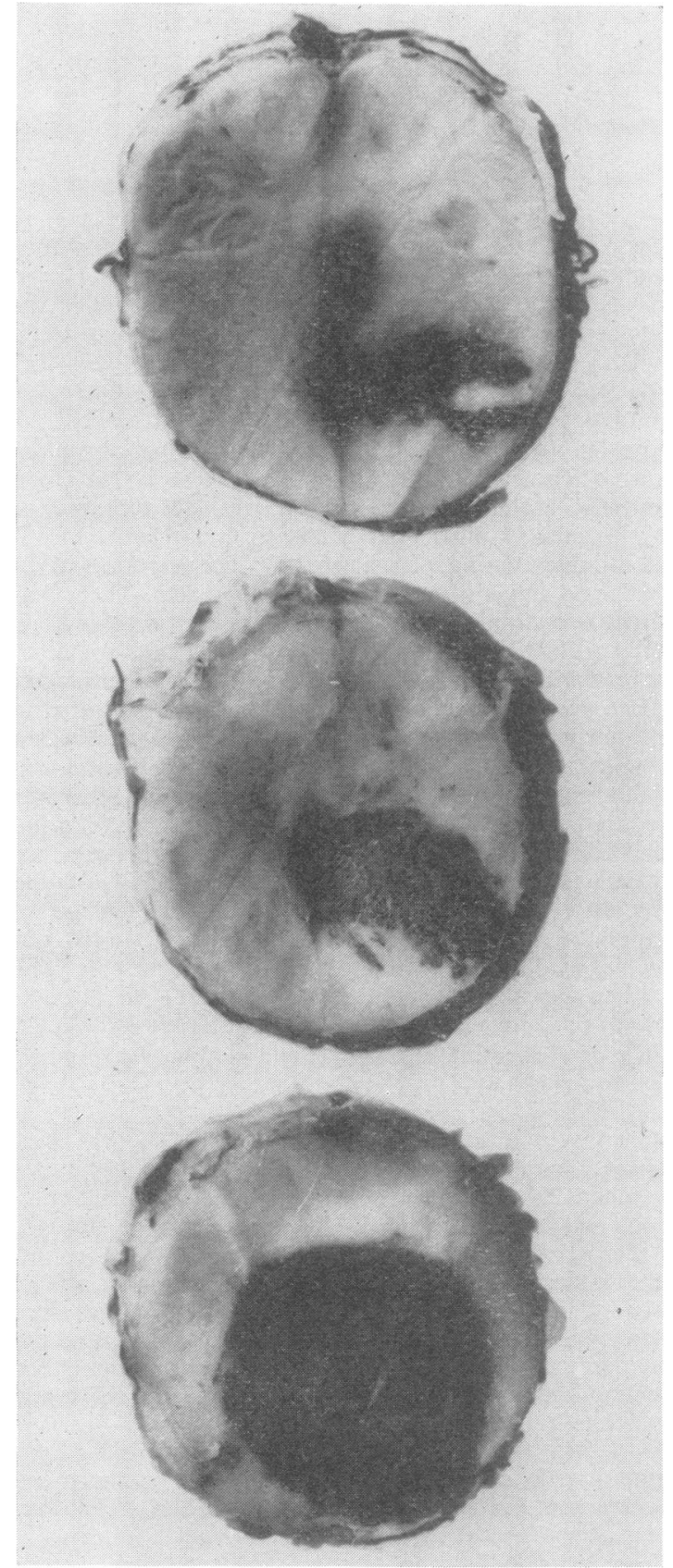

FIG. 1. Sections through the lower medulla in case 1 showing recent haemorrhage. posterior half of the cord. There was no haemorrhage into the ventricular system. Microscopic examination of the medulla showed a well-circumscribed recent haemorrhage at the margins of which were many smaller satellite petechial haemorrhages, many of them perivascular. In most instances details of the vessel wall were preserved but in a few there appeared to have been rupture of the vessels. The walls of many arterioles and small arteries in the medulla showed advanced hyalinization of the media, while in the more severely affected ones the intima was also involved (Fig. 2.)

Examination of the rest of the brain revealed no abnormality. The vessels of the circle of Willis showed moderately severe focal atherosclerosis. The basilar artery was the most severely affected but there was only minimal narrowing of the lumen of this vessel.

\section{CASE 2}

The patient was an 87-year-old woman who was admitted to the Royal Perth Hospital on 27 May 1967 having been found unconscious at the convalescent home where she lived, some three hours after the evening meal. No further details were available. She had been treated with digitalis for several years for control of congestive cardiac failure.

On admission she was drowsy but could be roused too obey simple commands. There was no spontaneous $\mathbb{\Phi}$ speech and, although she attempted to answer questions, $\frac{\stackrel{\mathbb{D}}{\mathrm{D}}}{\mathrm{O}}$ she was unable to speak. She responded to visual stimulio in all parts of both visual fields, but visual acuity could $\vec{C}$ not be accurately assessed and the presence of bilateralo cataracts did not allow an adequate fundoscopic exam-0 ination. The eyes were deviated to the left and she was unable to look to the right. The pupils were equal inf size and reacted to light, both directly and consensually and on accommodation. The corneal reflexes were present on both sides and there was no impairment of facial sensation. There was weakness of both upper and lower portions of the right side of the face and there were occasional myoclonic movements of the angle of the right side of the mouth. The palate failed to move on either side and a gag reflex was not obtained. She was unable to swallow the secretions which accumulated in the pharynx and which could be aspirated with no discomfort. There was no weakness of the sternomastoids or trapezii and the tongue protruded in the midline. The patient made spontaneous movements with all four limbs, but muscle power was reduced in the left arm and leg. Muscle tone was increased in the left arm and leg and the deep tendon reflexes were brisker on the left than on the right. The superficial abdominal reflexes were absent on both sides and there was a left-sided Babinski response. There was mild inco-ordination in the left arm and leg but no sensory abnormalities. She was afebrile, the pulse was 96 per minute and regular; blood pressure $180 / 100 \mathrm{~mm}$ $\mathrm{Hg}$; respiration was regular at a rate of 20 per minute. There was evidence of left ventricular hypertrophy and of mild congestive cardiac failure. A chest radiograph confirmed the presence of cardiomegaly, and an electrocardiogram showed left axis deviation, left ventricular hypertrophy, and ventricular ectopic beats. 


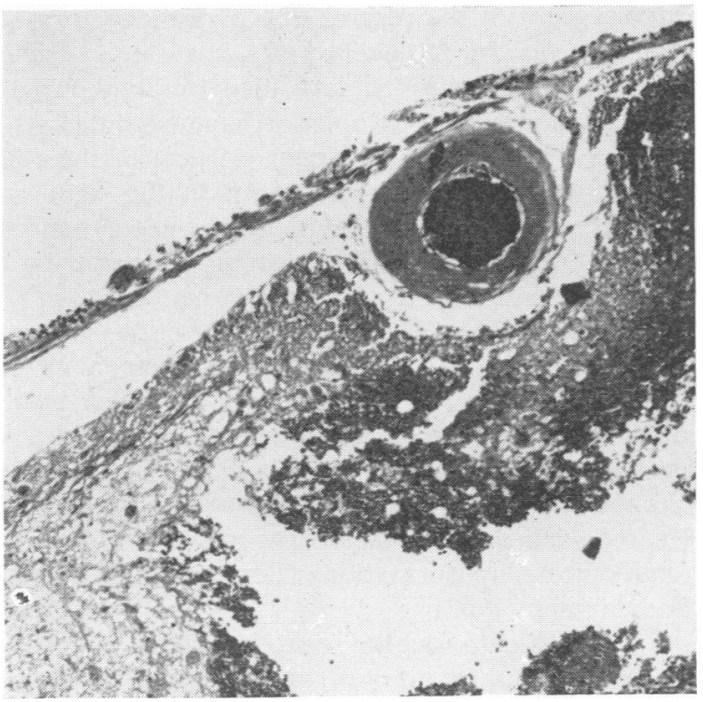

FIG. 2. Hyaline change in the wall of a small artery at the edge of the haemorrhage in case 1. Haematoxylin and eosin $\times 115$.

By the fourth hospital day the patient made attempts to speak but was dysarthric. She experienced considerable difficulty in swallowing both fluids and solids. Her neurological state remained unchanged and she died on the 21st hospital day.

NECROPSY REPORT At necropsy (B.E.) (A67.266) there was evidence of hypertensive cardiovascular disease and widespread atherosclerosis. The heart weighed $372 \mathrm{~g}$ and there was hypertrophy of the left ventricle. The kidneys weighed 78 and $82 \mathrm{~g}$. Microscopic examination showed widespread hyaline arteriosclerosis consistent with longstanding benign hypertension.

NEUROPATHOLOGICAL FINDINGS A recent haemorrhage was present on the right side of the basis pontis and extended into the upper medulla on the right side (Fig. 3). In its rostral portion it occupied most of the medial tegmentum and dorsal part of the basis pontis on the right side. At a more caudal level it was situated more ventrally and involved the medial portion of the right inferior olivary nucleus, the right pyramidal tract, and adjacent arcuate nuclei in the medulla. Microscopic examination showed that the haemorrhage was well circumscribed from the surrounding parenchyma, which showed vacuolation, many axonal swellings, and numbers of pleomorphic microglia. Around the margin of the haemorrhage were many capillaries and macrophages which contained lipid material and haemosiderin. Mononuclear cells were also present in the outermost layers of the haematoma (Fig. 4). The media of many arterioles and small arteries presented a homogeneous eosinophilic appearance.

Examination of the rest of the brain showed an old area

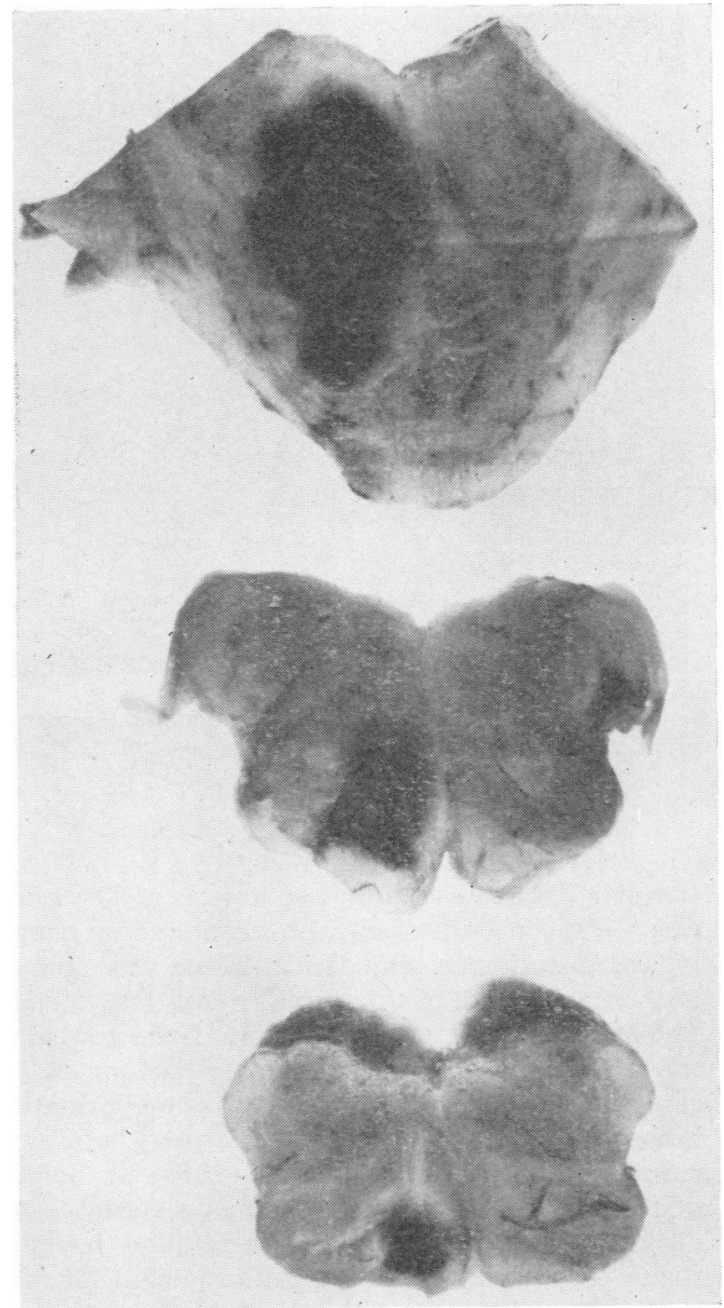

FIG. 3. Sections through the lower pons and upper medulla in case 2 showing the extent of the haemorrhage.

of infarction in the left putamen and external capsule, several petechial haemorrhages in the right putamen, and small lacunar areas of infarction were present in the left hippocampus and in the white matter of the left cerebellar hemisphere. The basal arteries were severely atheromatous with irregular fusiform dilatation of the vertebral, basilar, and internal carotid arteries.

\section{DISCUSSION}

The first patient was hypertensive and the haemorrhage occurred during a period of inadequately controlled anticoagulant therapy for ischaemic heart disease. In the second case there was no history of hypertension but evidence of hypertensive cardio- 


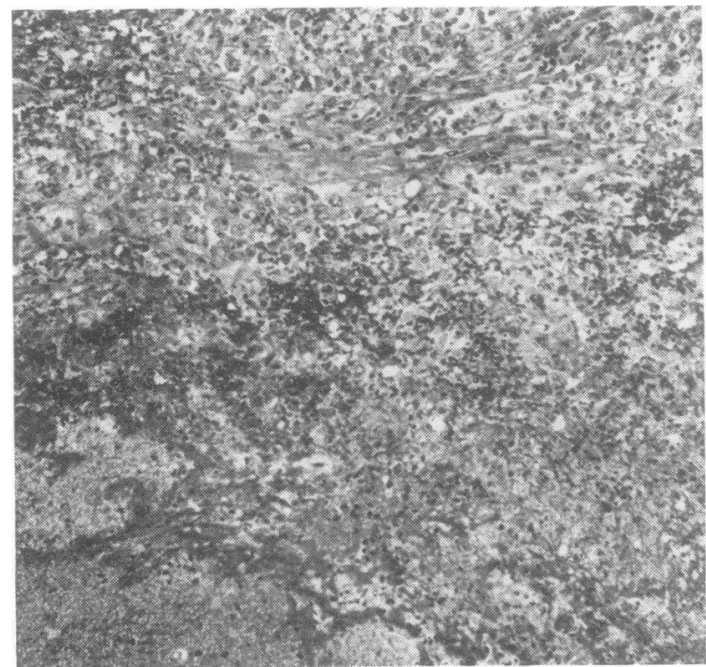

FIG. 4. Macrophage and capillary reaction at the margin of the haemorrhage in case 2. Haematoxylin and eosin $\times 118$.

vascular disease was found at necropsy. The onset was abrupt in both cases with no preceding neurological disturbance, and the outcome was fatal in both after periods of three and 21 days respectively. The cases reported in the literature have likewise all run a rapidly fatal course, although in one of the cases associated with an angiomatous malformation bulbar signs were first manifest five years before the eventual fatal rupture (Bergman, 1950). In neither of the present cases was the diagnosis established during life. Although some intracranial haemorrhagic complication of the anticoagulant therapy was suspected in case 1 the diagnosis of medullary haemorrhage was not made. In the second case a diagnosis of ponto-medullary infarction due to vertebrobasilar insufficiency was made during life. Differentiation from the latter far commoner condition may be possible if there is an adequate history of any preceding neurological episodes and of the onset and progression of the neurological deficits.

In the first case the haemorrhage was situated in the lower portion of the medulla and extended into the upper cervical cord. In its rostral portion it occupied the posterior part of the left side of the medulla and involved the gracile and cuneate nuclei, accounting for the loss of vibration and position sense in the left limbs. Involvement of the spinal tract and nucleus of the trigeminal nerve and spinothalamic tract was responsible for the subjective sensory manifestations on the right side of the body.
Involvement of the decussating pyramidal fibres on the right side by the haemorrhage at a more caudal level accounted for the left-sided pyramidal signs. In the second case the haemorrhage extended from the lower pons into the upper portion of the right side of the medulla. Involvement of the right side of the pons resulted in right lower motor neurone facial paralysis, paralysis of conjugate movement of the eyes to the right, and crossed hemiparesis. The anarthria, dysphagia, and palatal paralysis were attributed to a combination of involvement of the right nucleus ambiguus and vagal nucleus in the medulla and bilateral supranuclear pyramidal tract involvement. In neither case had the haemorrhage ruptured into the ventricular system, as occurred in the reported cases of haemorrhage due to rupture of angiomatous malformations (Bergman, 1950; Bosch and Janssen, 1962).

In neither case was the source of the haemorrhage obvious. In particular, no vascular malformation, large or cryptic, was found. There has been considerable controversy regarding the pathogenesis of primary intracerebral haemorrhage and several hypotheses have been put forward in the past. Thes $\omega$ have been summarized by several authors including Globus (1938), Mutlu et al. (1963), and Ross Russe $\rightarrow$ (1963). The original suggestion of Charcot an $\overline{8}$ 음 Bouchard (1868) that many such haemorrhagesresult from the rupture of small arterial aneurysm $\subseteq$ is now more widely accepted, particularly since the demonstration by Ross Russell (1963) that micros $\overrightarrow{0}$ aneurysms are found in many hypertensive patients particularly in the basal ganglia and thalamus, parts of the brain where there are long penetrating arteries and which are common sites for primary intracerebral haemorrhage. Hyalinosis of arteriolar walls as described by Scholtz and Neito (1938) has also been implicated as the pathological basis for $\stackrel{\mathbb{Q}}{2}$ arterial rupture leading to cerebral haemorrhage. $\overrightarrow{\overrightarrow{0}}$ This change appears to be indistinguishable from 3 benign hypertensive arteriolosclerosis which was present in both of the present cases. Shwartz (1930) proposed that large haemorrhages may result from confluence of smaller petechial extravasations from $\frac{7}{0}$ vessels weakened by neurogenic spasm. The findings $:-$ of small perivascular haemorrhages at the margin 3 of the haematoma in case 1 suggest that this $\delta$ mechanism may have had some part to play in its formation.

The rarity of medullary haemorrhages must be $\frac{\text { 음 }}{D}$ related in some way to regional anatomical oro haemodynamic peculiarities. Mutlu et al. (1963) attached significance to the fact that most medullary or arteries run horizontally in contrast to those of the $N$ pons. They also suggest that the large cisterna magna N may protect the venous circulation from compression 
between the medulla and the base of the skull accounting for the rarity of secondary venous haemorrhages in the medulla. Façon et al. (1962) suggested that haemorrhages are commoner in the rostral portions of the brain-stem because of the fact that vascular malformations are much more common in this region. However, most cases of primary pontine haemorrhage are unassociated with vascular malformations and a more likely explanation is that microaneurysms are commoner in the pons which is supplied by long penetrating branches from the basilar artery (Cole and Yates, 1967). Unlike the pons the medulla does not have these long penetrating vessels of supply and this may account for its relative immunity to primary haemorrhage. Ross Russell (1963) in his microangiographic studies confined himself to examination of the striate arteries and did not comment on the vessels of the brain-stem. However, similar studies by Cole and Yates (1967) failed to demonstrate micro-aneurysms in the medulla, whereas they were relatively common in the pons. Further studies of the vasculature of the brain-stem may throw further light on the differential susceptibility of the pons and the medulla to primary haemorrhage and also on the role of the Charcot-Bouchard aneurysm in the pathogenesis of cerebral haemorrhage.

\section{SUMMARY}

Haemorrhage into the medulla oblongata is an uncommon occurrence. The clinical and pathological features of two cases of medullary haemorrhage are described with comments on possible pathogenetic mechanisms and possible reasons for the rarity of the condition.

We wish to thank Dr. J. B. Stokes for providing us with clinical details of case 1 . Professor R. E. J. ten Seldam performed the necropsy in this case. The photographs of the brain were prepared by Mr. R. van Raalte of the Department of Medical Illustrations of the Royal Perth Hospital and the photomicrographs by Mr. H. Upenieks, A.R.P.S. Dr. F. L. Mastaglia is in receipt of a Saw Medical Research Fellowship from the University of Western Australia.

\section{REFERENCES}

Barron, K. D., and Fergusson, G. (1959). Intracranial hemorrhage as a complication of anticoagulant therapy. Neurology (Minneap.), 9, 447-455.

Bergman, P. S. (1950). Hemangioma of the pons. Case report and review of the literature. J. Mt Sinai Hosp., 17, 119-131.

Bosch, K., and Janssen, W. (1962). Plotzlicher Tod Durch Intramedulläres Hämangiom. Dtsch. Z. ges. gerichtl. Med., 52, 571-577.

Charcot, J. M., and Bouchard, C. (1868). Nouvelles recherches sur la pathogenie de l'hemorrhagie cérébral. Arch. Physiol. norm. Path., 1, 110-123.

Cole, F. M., and Yates, P. O. (1967). The occurrence and significance of intracerebral micro-aneurysms. J. Path. Bact., 93, 393-411.

Façon, E., Schwarz, B., and Ionescu, S. (1962). Das Spontanhämatom des Hirnstamms. Wien. klin. Wschr., 74, 655-658.

Globus, J. H. (1938). Massive cerebral haemorrhage: its antecedent and precipitating factors. Res. Publ. Ass. nerv. ment., Dis., 18, 438-470.

Gross, S. W., and Ehrlich, W. (1940). Diagnosis and Treatment of Head Injuries. Paul B. Hoeber, New York and London.

Mutlu, N., Berry, R. G., and Alpers, B. J. (1963). Massive cerebral hemorrhage: clinical and pathological correlations. Arch. Neurol. (Chic.), 8, 644-661.

Pezzani, M., and D'Alessio, C. (1956). Le emorragie bulbari nell'anoxia fetale. Arch. ostet. Ginec., 61, 347-367.

Ross Russell, R. W. (1963). Observations on intracerebral aneurysms. Brain, 86, 425-441.

Scholtz, W., and Neito, D. (1938). Studien zur Pathologie der Hirngefässe: 1. Fibrose und Hyalinose. Z. ges. Neurol. Psychiat., 162, 675-693.

Schwartz, P. (1930). Die Arten der Schlaganfälle des Gehirns und ihre Entstehung. Springer-Verlag, Berlin. 